\title{
Surgical management of Giant paraclinoid internal carotid artery aneurysm management in Endovascular era
}

\author{
Prabhat Jha $M S^{1}$, Binit Kumar Jha $M S^{2}$, Manoj Bohara $P h D^{3}$, Rajiv Jha $M C^{4}$, Prakash Bista $M C h^{5}$ \\ ${ }^{1,2,4,5}$ National Neurosurgical Referral Center, National Academy of Medical Sciences, Kathmandu, Nepal, ${ }^{3}$ Stroke \& \\ Neurointerventional Surgery, HAMS Hospital, Kathmandu, Nepal
}

Date of submission: $3^{\text {rd }}$ October 2020

Date of acceptance: $15^{\text {th }}$ February 2021

Date of publication: $1^{\text {st }}$ March 2021

\begin{abstract}
Giant aneurysms are lesions with diameter greater than or equal to $25 \mathrm{~mm}$. They are uncommon lesions presenting with features of mass effect and rarely rupture. Diagnosis is confirmed on angiography.

This case describes a young female with giant paraclinoid internal carotid artery aneurysm. The patient was managed with superficial temporal artery to middle cerebral artery bypass with aneurysm trapping.
\end{abstract}

Key words: Aneurysm trapping, Giant aneurysm, STA-MCA bypass

\section{Introduction}

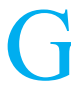
iant aneurysms are rare lesions with a high female prevalence. They usually occur in the fourth to sixth decades of life. Locksley et al have classified aneurysms that are $25 \mathrm{~mm}$ or greater as giant. ${ }^{1,2}$ They are associated with high rate of morbidity and mortality. ${ }^{3}$ The most common location is along the distribution of internal carotid artery. Degeneration of the muscular and elastic laminar layer of blood vessels, repeated intramural

Access this article online
Website: https://www.nepjol.info/index.php/NJN
DOI: https://doi.org/10.3126/njn.v18i1.31750
HOW TO CITE
Jha P, Jha BK, Bohara M, Jha R, Bista P. Surgical management of
Giant paraclinoid internal carotid artery aneurysm management
in Endovascular era. NJNS. 2021;18(1):61-65.

${ }^{1}$ ORCID id: 0000-0001-5504-5917

${ }^{2}$ ORCID id: 0000-0002-0844-2538

${ }^{3}$ ORCID id: 0000-0002-3157-7334

${ }^{4}$ ORCID id: 0000-0002-2830-7835

${ }^{5}$ ORCID id: 0000-0002-4811-6964

Address for correspondence:

Dr. Prabhat Jha,

MCh Neurosurgery Resident,

National Neurosurgical Referral Center,

National Academy of Medical Sciences, Kathmandu, Nepal

E-mail: prabhatkaushaljha@gmail.com

Phone: +977-9851208714

Copyright (C) 2021 Nepalese Society of Neurosurgeons (NESON)

ISSN: 1813-1948 (Print), 1813-1956 (Online)

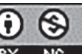

This work is licensed under a Creative Commons Attribution-Non Commercial 4.0 International License. hemorrhage within the aneurysm wall followed by thrombosis and neovascularization and trauma are believed to be involved in the pathogenesis of giant aneurysms. Smoking, hypertension, connective tissue disorders are the risk factors for these lesions. ${ }^{4}$ Most commonly they present with mass effects due to the compression of adjacent structures. Four vessel cerebral angiography is the gold standard for diagnosing these lesions. Management options include direct and indirect surgical procedures as well as endovascular procedures. ${ }^{5}$

\section{Case}

A 35 year old female from southern Nepal presented with history of severe headache associated with vomiting multiple episodes for 3 days. The patient also complained of blurring of vision following the headache. There was no history of fever, trauma, seizure, focal deficits, neck rigidity. Patient's bowel and bladder habits were normal. Her menstrual history was regular. Her past medical history was non-significant.

At presentation in emergency her vital signs were normal. Her Glasgow Coma Scale (GCS) was E3V4M6 $(13 / 15)$. There were no neurological deficits and her systemic examination were within normal limits.

Her biochemical and hematological parameters were within normal limits. Non-Contrast Computed tomography (NCCT) head showed hyperdense lesion in the sellar region (Figure 1). CT angiography of head was done which showed a giant aneurysm arising from the left paraclinoid segment of internal carotid artery (ICA). Anterior cerebral artery (ACA) and middle cerebral artery (MCA) were compressed by the aneurysm (Figure 1). Digital subtraction angiography (DSA) was done to 


\section{Jha et al}

better analyze the aneurysm and plan the best treatment option. DSA demonstrated a giant left paraclinoid internal carotid artery aneurysm. ACA and MCA on left side were compressed by the aneurysm. No evidence of thrombus, aneurysm, fistula was seen. Right internal carotid artery with its branches, bilateral vertebral arteries, bilateral posterior cerebral arteries and basilar artery were normal with no evidence of filling defect. DSA showed that there was a good collateral circulation through the circle of Willis as evaluated by cross compression during angiography as well as by clinical determination of the tolerance to the carotid occlusion. Therefore, the patient has been accepted as a good candidate for proximal carotid artery ligation (Figure 1).

The patient was planned for surgery. The procedure planned was superficial temporal artery (STA) to MCA bypass followed by aneurysm trapping. Preoperative central line, arterial line, Foley catheterization was done. Patient was positioned on a Mayfield horseshoe with $45^{\circ}$ head tilt to right. Incision was marked in the cervical region for ICA control and pterional craniotomy was planned.
The ICA on left side was identified and controlled. Then the superficial temporal artery (STA) on left side was dissected along with its frontal and parietal branches. Craniotomy was done and the cortical (M4) segment of MCA was dissected. End to side anastomosis between the parietal branch of STA and M4 segment was done. Left sylvian fissure dissection was done. Then the aneurysm was identified. The aneurysm was approximately 5 x 5 $\mathrm{cm}$ in size. Temporary clip was applied to internal carotid artery on left side and collapse of aneurysm observed. Ligation of internal carotid artery was done in neck followed by clipping of the distal segment of aneurysm (Figure 2,3). Indocyanine green was injected which showed no flow into the aneurysm. The patient was extubated on postoperative day 1. Post-operative CT head showed thrombosed aneurysm in sellar region (Figure 4). Patient had postoperative motor aphasia.

At 3 months follow up there were no symptoms and focal neurological deficits. DSA showed complete occlusion of the aneurysm with flow through left ACA and MCA. Patient was producing words (Figure 4, 5).

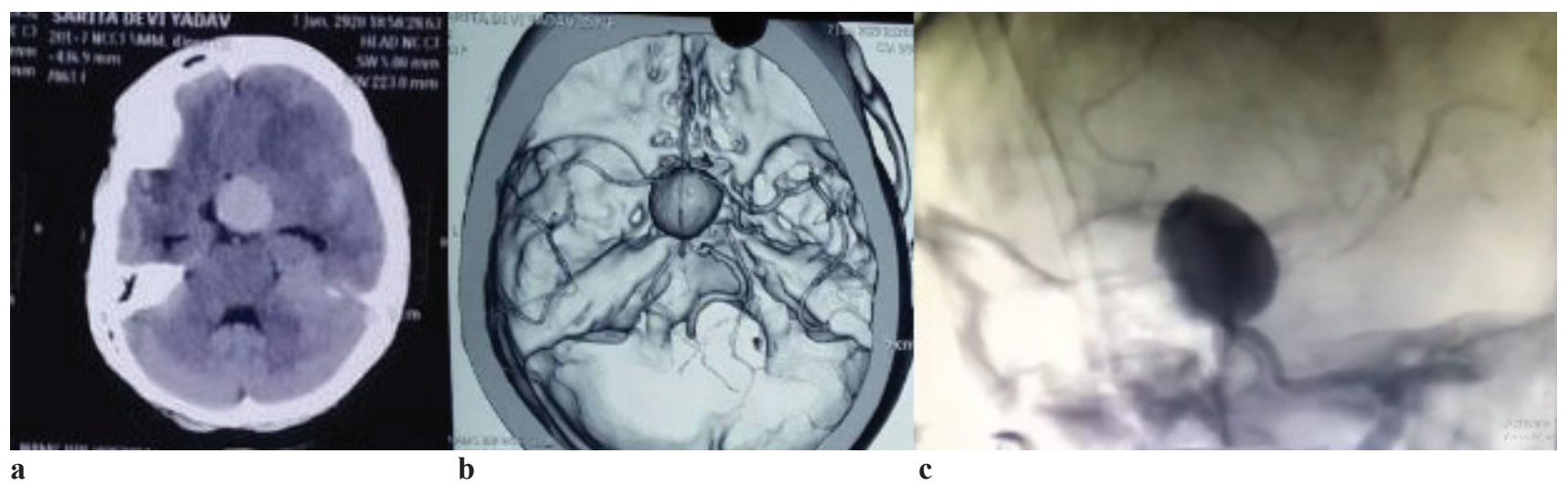

a b

Figure 1:(a) Preoperative NCCT head showing hyperdense lesion in sellar region, (b) Reconstructed CT angiography showing a left sided giant paraclinoid internal carotid artery aneurysm, (c) Digital subtraction angiography showing giant aneurysm

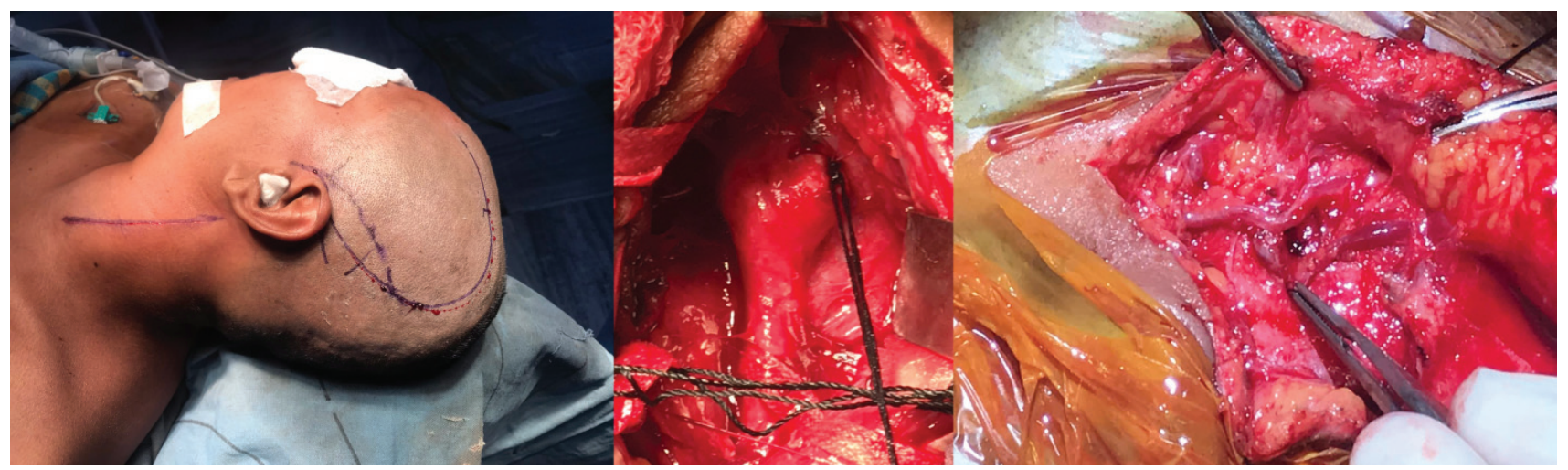

Figure 2: Intraoperative incision marking, internal carotid artery isolated in neck, Superficial temporal artery to middle cerebral artery bypass 


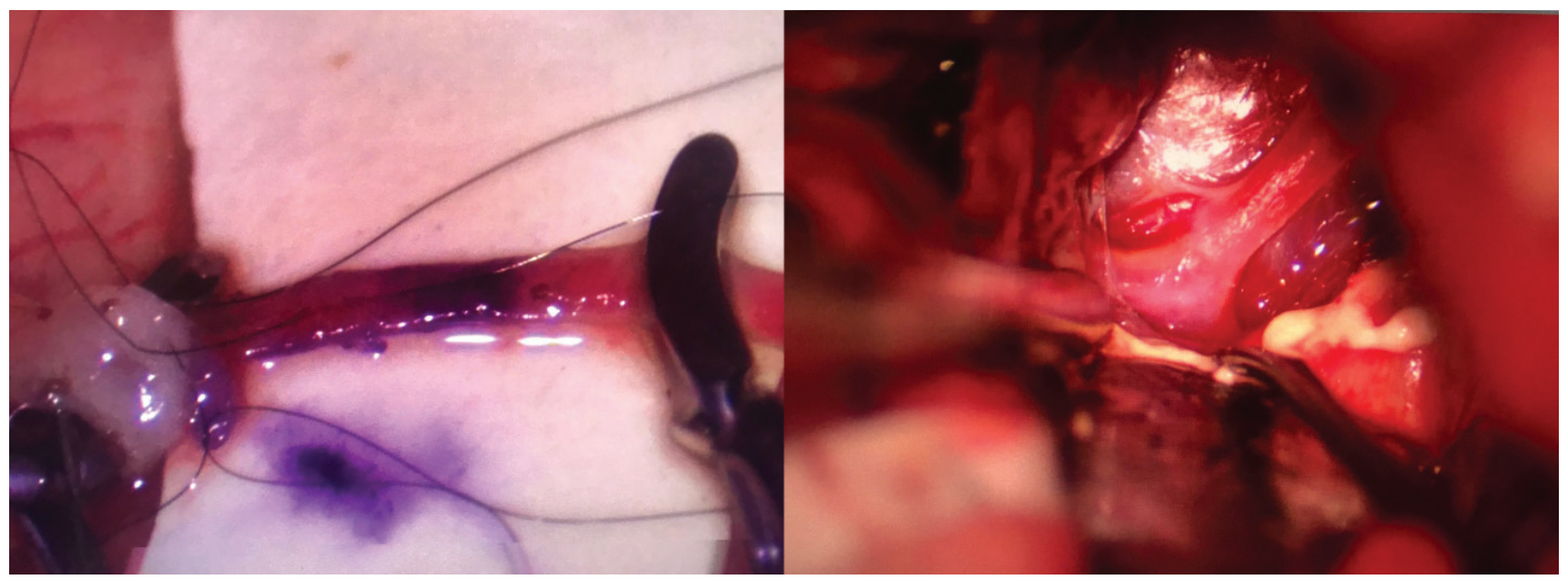

Figure 3: STA-MCA bypass, view of giant aneurysm intraoperatively

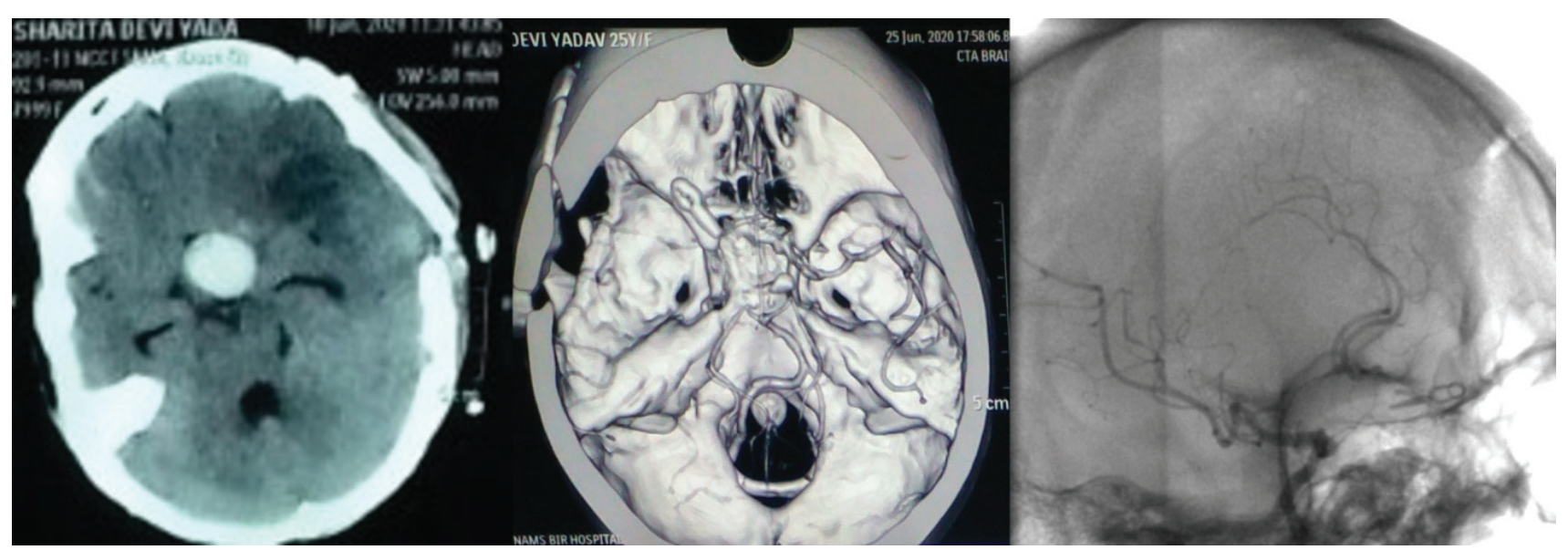

Figure 4 (a) Immediate postoperative CT head, (b) Reconstructed CT angiography at 3 months showing complete occlusion of aneurysm, (c) DSA at 3 months showing complete occlusion of aneurysm

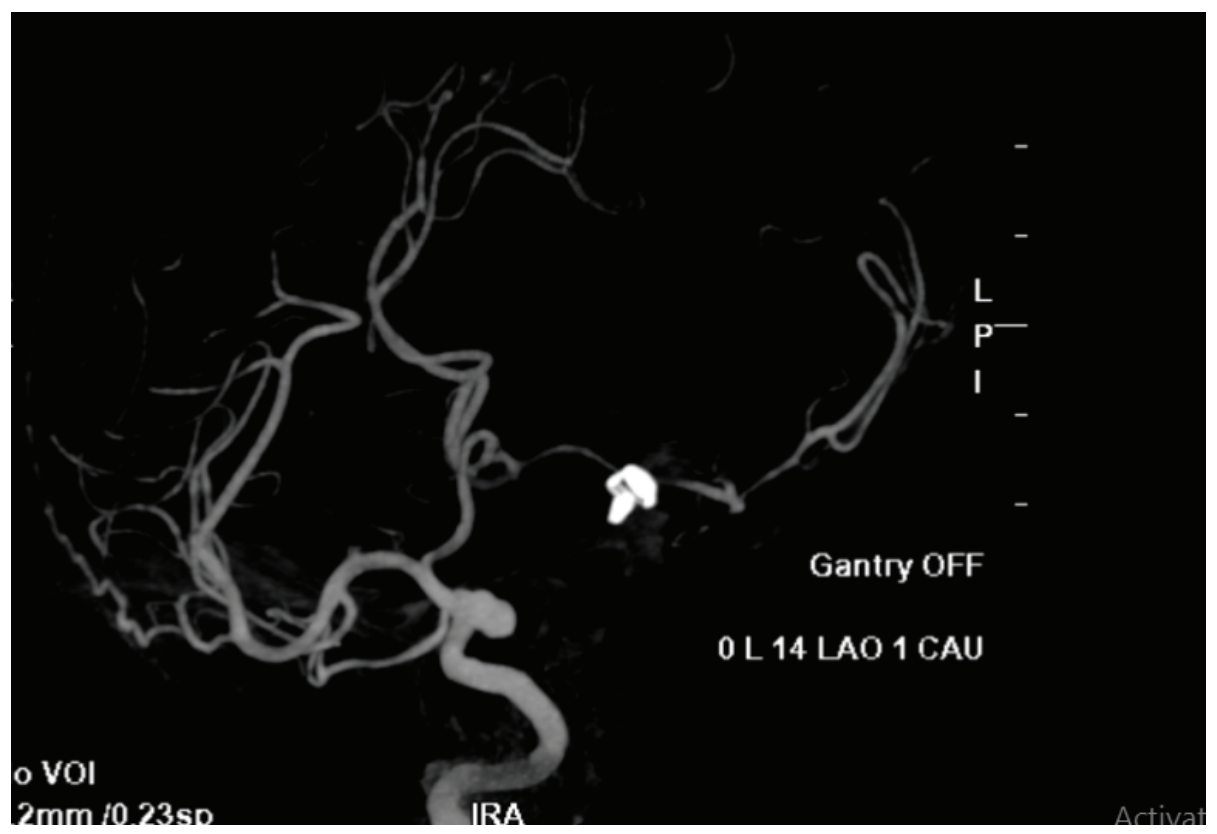

Figure 5: DSA at 3 months showing flow through the ACA and MCA on left side and absent intracranial trapped portion of left ICA 


\section{Discussion}

Giant aneurysms represent $2 \%$ to $5 \%$ of all intracranial aneurysms. ${ }^{6}$ As with other aneurysms, giant aneurysms have a female prevalence, although Kodama and Suzuki found an equal sex distribution. ${ }^{7}$ Most patients become symptomatic in the fourth through sixth decades of life. ${ }^{8}$ Our patient is also a female in third decade of life.

Hutchinson described the first giant intracranial aneurysm in 1875, which was diagnosed by an audible bruit. Based on 6368 cases in a cooperative study, Locksley classified aneurysms that were $25 \mathrm{~mm}$ or greater as giant and observed a high rate of morbidity and mortality associated with these lesions. ${ }^{1,9}$ Giant aneurysms are found in all locations throughout the intracranial vasculature. In general, $34 \%$ to $67 \%$ of giant intracranial aneurysms are associated with the ICA, $11 \%$ to $40 \%$ with the anterior cerebral artery (ACA) and middle cerebral artery (MCA), and $13 \%$ to $56 \%$ with the vertebral and basilar arteries. ${ }^{10-14}$ Our patient had a paraclinoid aneurysm approximately 5 $\mathrm{cm}$ in size.

Giant aneurysms can develop due to defects in the medial layer of blood vessel, due to repeated intramural hemorrhage within the aneurysm wall followed by thrombus formation, de novo. ${ }^{15}$ Numerous factors have been linked to the formation and rupture of aneurysms, including female sex, age, hypertension, connective tissue disease, and smoking. ${ }^{15}$ No specific risk factor was identified in our case.

Most patients develop signs and symptoms related to mass effect (i.e., brainstem compression, cranial nerve palsy, and seizure) depending upon the location and up to one third may present with SAH. ${ }^{1-5}$ Patients may present with headache, thromboembolism to distal vascular territories. The annual risk of rupture is correlated with increasing aneurysm size. ${ }^{16}$

Four-vessel cerebral angiography has long been considered the "gold standard" for the diagnosis of cerebral aneurysms, providing information about an aneurysm's location, anatomy, adjacent branch vessels, collateral circulation, and distal cerebral perfusion and another intracranial aneurysm. ${ }^{15,16}$ Our patient was also diagnosed on cerebral angiography. Beside DSA is useful to determine cross flow along circle of Willis. This helps to determine if internal carotid artery can be ligated at neck. CT helps in defining the outer dimensions of an aneurysm if the aneurysm is thrombotic. Besides the relationship of the aneurysm to skull base can be studied in CT. MRI is useful to identify the compressive effect on adjacent brain and neural structures. ${ }^{14,15}$

Giant cerebral aneurysms left untreated have a mortality rate up to $68 \%$ within 2 years and up to $85 \%$ within 5 years of diagnosis. ${ }^{16}$ Patients presenting with SAH should be managed according to accepted principles and protocols. Conservative management is not recommended unless patient is at a high risk for surgical intervention. Different direct and indirect surgical procedures have been described. The accepted method of treatment is occlusion of ICA with or without bypass. Endovascular interventions have less morbidity than open procedures. Increasingly, endovascular techniques are being combined with operative techniques to improve outcomes. ${ }^{15,16}$ Direct surgical approach to the internal carotid artery giant aneurysm and its resection has been described. In some cases, resection of the aneurysm with reconstruction of internal carotid artery is done. But the direct surgical exposure is difficult. So, ligation of the ipsilateral internal carotid artery in neck is considered an option with the goal of decreasing intra-arterial pressure in the distal segment and cause aneurysm thrombosis. But it can cause ischemic complications. The carotid occlusion can be instituted either acutely or gradually with most authors favoring a gradual occlusion over a number of days.

The management options would depend on the expertise available specially for endovascular procedure and the anatomy of the aneurysm. Our patient was managed with STA- MCA bypass with aneurysm trapping. STA-MCA bypass with aneurysm trapping was chosen in our case because the aneurysm was very large and we initially thought that cross flow would be necessary. Besides proximal occlusion of aneurysm was deemed quite difficult because of the location. So, trapping was done in neck

\section{Conclusion}

Giant intracranial aneurysms are rare lesions with high risks of rupture. Surgical bypass with aneurysm trapping is one of the methods of management.

\section{Conflict of Interest: None}

Source(s) of support: None

\section{Abbreviations}
ACA- anterior cerebral artery
CT- computed tomography
DSA- digital subtraction angiography
GCS- Glasgow coma scale
ICA- internal carotid artery
MCA- middle cerebral artery
MRI- magnetic resonance imaging
SAH- subarachnoid hemorrhage
STA- superficial temporal artery 


\section{Giant paraclinoid internal carotid artery aneurysm}

\section{References}

1. Locksley HB, Sahs AL, Sandler R. Report on the cooperative study of intracranial aneurysms and subarachnoid hemorrhage. 3. Subarachnoid hemorrhage unrelated to intracranial aneurysm and A-V malformation. A study of associated diseases and prognosis. J Neurosurg. 1966 Jun;24(6):103456. https://doi.org/10.3171/jns.1966.24.6.1034

2. Sahs AL, Perret G, Locksley HB, Nishioka H. Intracranial Aneurysms and Subarachnoid Hemorrhage: A Cooperative Study. Philadelphia, PA: Lippincott; 1969.

3. Wiebers DO, Whisnant JP, Huston J 3rd, Meissner I, Brown RD Jr, Piepgras DG, Forbes GS, Thielen K, Nichols D, O’Fallon WM, Peacock J, Jaeger L, Kassell NF, Kongable-Beckman GL, Torner JC; International Study of Unruptured Intracranial Aneurysms Investigators. Unruptured intracranial aneurysms: natural history, clinical outcome, and risks of surgical and endovascular treatment. Lancet. 2003 Jul 12;362(9378):103-10. https:/doi. org/10.1016/s0140-6736(03)13860-3

4. Krings T, Alvarez H, Reinacher P, et al. Growth and rupture mechanism of partially thrombosed aneurysms. Interv Neuroradiol. 2007;13(2):117-126. https://doi.org/10.1177/159101990701300201

5. Kato Y, Sano H, Imizu S, Yoneda M, Viral M, Nagata J, Kanno T. Surgical strategies for treatment of giant or large intracranial aneurysms: our experience with 139 cases. Minim Invasive Neurosurg. 2003 Dec;46(6):339-43. https://doi. org/10.1055/s-2003-812499

6. Morley TP, Barr HW. Giant intracranial aneurysms: diagnosis, course, and management. Clin Neurosurg. 1969;16:73-94. https://doi.org/10.1093/ neurosurgery/16.cn_suppl_1.73
7. Kodama N, Suzuki J. Surgical treatment of giant aneurysms. Neurosurg Rev. 1982;5:155-160.

8. Atkinson JLD, Piepgras DG. Giant aneurysms: supratentorial. In: Carter LP, Spetzler RF, eds. Neurovascular Surgery. New York: McGraw-Hill; 1995:815-828.

9. Hutchinson J. Aneurysms of the internal carotid within the skull diagnosed 11 years before patient's death: spontaneous cure. Trans Clin Soc (London). 1875;8:127.

10. Barrow DL, Alleyne C. Natural history of giant intracranial aneurysms and indications for intervention. Clin Neurosurg. 1995;42:214-44. PMID: 8846594.

11. Hosobuchi Y. Giant intracranial aneurysms. In: Wilkins RH, Rengachary SS, eds. Neurosurgery. New York: McGraw-Hill; 1985: 1404-1414.

12. Lawton MT, Spetzler RF. Surgical management of giant intracranial aneurysms: experience with 171 patients. Clin Neurosurg. 1995;42: 245-266.

13. Symon L, Vajda J. Surgical experiences with giant intracranial aneurysms. J Neurosurg. 1984;61:10091028.

14. Yasargil MG. Giant intracranial aneurysms. In: Yasargil MG, ed. Microneurosurgery. Vol. II. Clinical Considerations, Surgery of the Intracranial Aneurysms and Results. New York: Thieme-Stratton; 1984: 296-304.

15. Koyama S, Kotani A, Sasaki J. Giant basilar artery aneurysm with intramural hemorrhage and then disastrous hemorrhage: case report. Neurosurgery. 1996;39:174-177.

16. Ausman JI, Diaz FG, Sadasivan B, et al. Giant intracranial aneurysm surgery: the role of microvascular reconstruction. Surg Neurol. 1990;34:8-15. 